\title{
The impact of tinnitus upon cognition in adults: A systematic review
}

\author{
Susan Tegg-Quinn ${ }^{1,2}$, Rebecca J. Bennett ${ }^{1,2}$, Robert H. Eikelboom ${ }^{1,2,3}$ \& David M. Baguley ${ }^{4,5}$ \\ ${ }^{1}$ Ear Science Institute Australia, Subiaco, Perth, Australia, ${ }^{2}$ Ear Sciences Centre, School of Surgery, The University of Western Australia, \\ Nedlands, Perth, Australia, ${ }^{3}$ Department of Speech-Language Pathology and Audiology, University of Pretoria, Pretoria, South Africa, \\ ${ }^{4}$ Department of Audiology and Otolaryngology, Addenbrooke's Hospital, Cambridge, UK, and ${ }^{5}$ Department of Audiology, Anglia Ruskin \\ University, Cambridge, UK
}

Correspondence: Susan Tegg-Quinn, Ear Science Institute Australia, Suite 1, Level 2, 1 Salvado Rd, Subiaco, WA 6008, Australia. E-mail: susan.tegg-quinn@earscience.org.au

\begin{abstract}
Objective: To systematically review and analyse experimental outcomes of studies exploring the impact of tinnitus upon cognitive function and their implications for clinical management of invasive tinnitus. Design: A systematic and descriptive review. Study sample: Eighteen studies were identified investigating the impact of tinnitus on cognitive function. Results: The 18 studies evaluated cognitive function using 24 different objective behavioural tests, nine electrophysiological recordings, one oculomotor test, and one self-report questionnaire. The studies spanned 18 years and revealed numerous interactions potentially contributing to the cognitive difficulties frequently reported by people with invasive tinnitus. The studies indicate a clear association between tinnitus and aspects of cognitive function, specifically the executive control of attention. Conclusions: Tinnitus impairs cognitive function by way of impact upon executive control of attention. Clinical management of patients reporting tinnitus and cognitive difficulties requires an understanding of the reciprocal relationship between tinnitus and cognitive function, with additive effects of anxiety, depression, and somatic cognitive bias. Further study is required to establish the impact of advancing age, hearing loss, anxiety, depression tinnitus duration, and distress upon cognitive function in people with invasive tinnitus.
\end{abstract}

Key Words: Tinnitus, cognition, systematic review

Subjective tinnitus describes the perception of sound in the absence of external stimuli (Searchfield, 2014). It may be experienced transiently as a spontaneous auditory perception, acutely resolving after a few minutes, up to several weeks, or it may become a chronic and debilitating symptom (Hall et al, 2011; Hoare et al, 2014). Chronic tinnitus can be episodic or continual and is characterized by episodes of tinnitus experienced for five minutes or longer for at least three months (Hall et al, 2011). In a recent study of the prevalence of tinnitus and the relationship with neuroticism approximately $16 \%$ of the 172,621 participants aged $40-69$ years reported tinnitus, with $3.8 \%$ of those with tinnitus describing it as troublesome (McCormack et al, 2014). The disparity between these two figures highlights the individual and heterogeneous nature of experiencing tinnitus.

Cognitive function encompasses the capacity for attention, concentration, use of working memory and information processing (Andersson \& McKenna, 2006). It is inextricably intertwined in the undertaking of a plethora of daily activities and experience of life through aspects such as thoughts, emotions, sensations, communication, perception, and reasoning (Andersson \& McKenna, 2006). Impaired cognitive function can have significant consequences for an individual's quality of life (Andersson, 2002; Westin et al, 2008). An association between tinnitus and reports of cognitive difficulties has long been noted, and increasingly, the reciprocal relationship between cognitive function to the experience of tinnitus, and the influence of tinnitus upon cognitive function is becoming apparent.

Contributions from the auditory cortex and non-auditory regions (Lanting et al, 2009) of the brain associated with attention and emotion to the perception of tinnitus and associated distress are being elucidated (Shulman et al, 1994; Rauschecker et al, 2010; Araneda et al, 2015). No longer is tinnitus thought to arise solely from the peripheral auditory structures. Damage to structures within the cochlear as a result of insult from factors such as noise exposure or aging may initiate processes associated with the development of tinnitus. These processes include alteration to the synchrony and spontaneous firing rates along the auditory nerve, 


\section{Abbreviations \\ ASSR Auditory steady state response \\ ERP Event related potential \\ MMSE Mini mental state examination \\ PRISMA Preferred reporting items for systematic reviews and meta-analyses}

alterations to the release, binding, and uptake of neurotransmitters (Potashner et al, 1997) as well as the development of neural hyperactivity along the auditory brainstem (Vogler et al, 2011) and central auditory pathway (Mulders \& Robertson, 2009). These alterations along the brainstem and midbrain are thought to be followed by neuroplastic reorganization within the auditory cortex and thalamus (Rauschecker et al, 2010) and accompanied by changes to both the structure and function of areas including the limbic and paralimbic areas, prefrontal cortex, hippocampus, and amygdala (Shulman et al, 1994; Rauschecker et al, 2010; Pace \& Zhang, 2013). Additionally, changes within the thalamus and inferior colliculus (Melcher et al, 2000; Rauschecker et al, 2010; Mahoney et al, 2011) may influence the impact of tinnitus upon cognitive function (Araneda et al, 2015). Neuroanatomical changes associated with tinnitus may impair the efficiency of cognitive processing and negatively impact on both selective and divided attention through impact upon the executive control of attention (Araneda et al, 2015). Similarly, alterations to structures associated with auditory attention (De Ridder et al, 2011) and efferent structures within the subcallosal region (Rauschecker et al, 2010), may inhibit the central noise-cancelling pathways or inhibitory gating mechanisms and contribute to the awareness of the tinnitus percept (Rauschecker et al, 2010; De Ridder et al, 2014). Such changes would contribute to a negative reciprocal relationship between perception of tinnitus and cognitive function. Although several studies have investigated the effect of tinnitus on cognitive function, debate continues over whether tinnitus is associated with an impact upon working memory, a general reduction of processing speed, a generalized depletion of resources for attention or through impairment of specific subcomponents of attention (Mohamad et al, 2015).

The purpose of this review was to critically appraise studies investigating the impact of tinnitus upon cognitive function, building on the work of two previous reviews. Andersson and McKenna (2006) in a selective review of 11 studies, concluded that tinnitus was likely to impair cognitive function, tinnitus may impair performance of some cognitive tasks and people with tinnitus may have some cognitive bias. Mohamad and colleagues (Mohamad et al, 2015) reviewed nine studies and considered that tinnitus adversely affects executive attention, but that evidence regarding the impact of working memory and selective attention was mixed. The aim of this study was to facilitate accurate informational counselling, assist effective rehabilitation, and to provide a framework for future research.

\section{Methods}

This study followed the preferred reporting items for systematic reviews and meta-analyses (PRISMA) guidelines (Moher et al, 2009). The PRISMA guidelines were derived from the PRISMA statement and involve 27 guidelines developed which are used to establish evidence-based standards for both undertaking and reporting systematic reviews and meta-analyses (Liberati et al, 2009; Panic et al, 2013). The six NHMRC levels of evidence classify how effectively different study designs address the research question and minimize the risk of bias (Coleman et al, 2005). A descriptive analysis, quality appraisal and review of all included studies was undertaken and presented (Mokkink et al, 2009).

\section{Search strategy}

A literature search using the keywords (tinnitus) and (cognition OR memory OR attention OR concentration OR cognitive function OR mental activity) NOT (infant OR child OR adolescent OR paediatric OR animal OR balance OR hyperacusis OR implant OR pharmaceutical OR drugs) was undertaken in the PubMed, MEDLINE, CINHAL, EMBASE, and Scopus databases in November 2014. The search terms were deliberately broad and no date restriction was applied. Additional articles were obtained through hand searching the references of studies identified during the initial search.

\section{Selection criteria}

Abstracts for all articles identified during the electronic and hand searches were retrieved and reviewed against the inclusion criteria by a single researcher. Studies were excluded if it was apparent from the abstract that they did not meet the inclusion criteria: experimental studies published in English in peer reviewed journals whose primary objective was to assess the impact of tinnitus upon cognitive function in an adult population. The studies had to evaluate cognitive function using a clearly described, repeatable behavioural test, such as validated surveys or established diagnostic tests and include a control group.

Full texts for studies identified were reviewed independently by two of the authors against the inclusion criteria. Both were blinded to the other's recommendations until the process was completed. In the absence of a universally agreed measure of tinnitus, no restrictions were placed on the method of tinnitus evaluation. Any disagreements regarding inclusion or data extraction were discussed and a consensus was established.

\section{Data extraction}

Descriptive data extraction was undertaken independently by two blinded authors for the final 18 studies. The descriptive data extracted included the type of study, the National Health and Medical Research Council (NHMRC) levels of evidence (Coleman et al, 2005), cohort descriptions (including age and gender), tinnitus descriptions, tinnitus distress, audiometric data, cognitive aspect studied, anxiety and depression measures, and the studies' conclusions.

Given the significant heterogeneity between studies in terms of tinnitus descriptions, cognitive tests employed and subject characteristics, a primarily narrative systematic review and analysis was employed.

\section{Results}

The database search produced 2236 studies (including duplicates), and a further 14 studies were identified by hand searching. The initial inclusion process yielded 83 unique studies published from 1996 to 2014. Of these, 65 studies were excluded, leaving 18 studies (Figure 1).

All studies in the review were case-control studies with NHMRC Level III (2) (Coleman et al, 2005). 


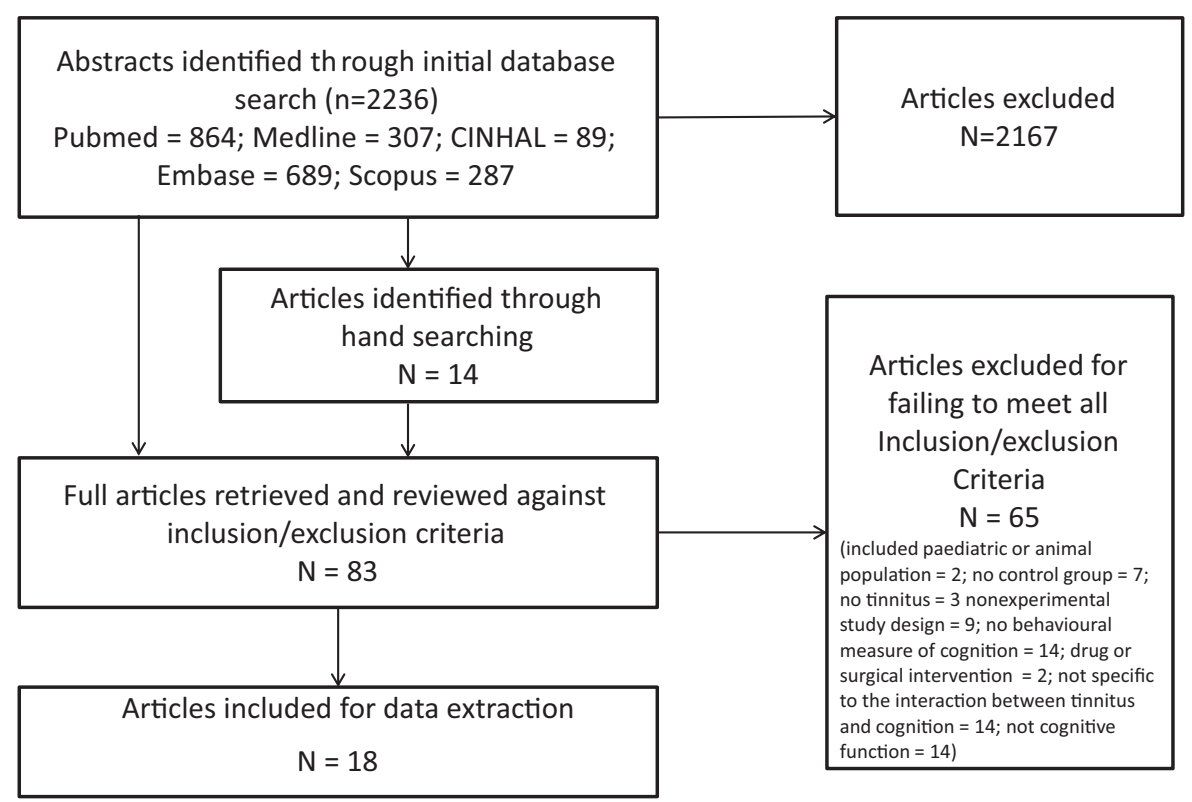

Figure 1. PRISMA Flow Chart for the identification of studies included in the systematic review.

Descriptions of cohorts' age

Two studies (Alam et al, 2012; Paul et al, 2014) reported neither the participants' mean ages and/or age ranges.

Ten studies matched their test and control groups for age or the difference was not statistically significant (Andersson et al, 2000, 2009, 2013; Dornhoffer et al, 2006; Rossiter et al, 2006; Stevens et al, 2007; Muluk et al, 2010; Gabr et al, 2011; Heeren et al, 2014; Jackson et al, 2014). These studies implicated an impact of tinnitus upon cognitive function. A negative impact of tinnitus upon cognition was concluded by the six studies where tinnitus subjects had older mean ages than control subjects (Jacobson et al, 1996; Andersson, 2002; Cuny et al, 2004; Andersson et al, 2005; Delb et al, 2008; Alam et al, 2012). However, none of these six studies commented on whether the age difference was statistically significant or if age was a confounding variable. Hallam et al (2004) noted a significant age difference between the groups in their study and concluded that the effects of tinnitus upon cognitive function may be subtle. Subject age has the potential to act as a confounding variable and was not well addressed. However, due to the studies' heterogeneity, it is impossible to isolate the impact age upon the results obtained.

\section{Cognition and electrophysiological indices}

The 18 studies used 24 different behavioural tests, nine different electrophysiological recordings, and one set of oculomotor tests to assess cognitive function (Table 1). Heeren et al (2014) employed a test specifically investigating the interaction between different attention networks. Three studies employed predetermined combinations of tests to assess either multiple aspects of cognitive function or the interaction between different networks (Muluk et al, 2010; Gabr et al, 2011; Heeren et al, 2014). Ten studies used multiple tests to assess different aspects of cognitive function, however, the rationale for the combinations were not clearly described. Only two studies justified their specific choice of test (Gabr et al, 2011; Heeren et al, 2014). Four studies used event related potential (ERP) recordings (Tables $1 \& 2$ ). Thirteen studies concluded that tinnitus had an impact upon cognitive function, however, only five quantified the degree of impairment: one as small (Cuny et al, 2004), two as subtle (Hallam et al, 2004; Rossiter et al, 2006), one as minor (Gabr et al, 2011), and one as significant (Dornhoffer et al, 2006). Two studies commented that result variation complicated quantifying the cognitive impact (Andersson et al, 2000; Andersson, 2002).

\section{Measures of tinnitus and tinnitus description}

Fourteen studies used seven different self-report tinnitus assessment scales to measure tinnitus distress. The tinnitus reaction questionnaire (Wilson et al, 1991) was the most frequently used (by five); the tinnitus questionnaire (Hallam, 1996) was used by three, and the subjective tinnitus severity scale (Halford \& Anderson, 1991) was used by two studies. Six studies used psychoacoustic measures including tinnitus pitch, loudness, minimum masking levels (MML), uncomfortable loudness levels, and residual inhibition to describe the tinnitus experienced. However, no single psychoacoustic measure was employed by all six studies. Six studies included a measure of tinnitus duration from time of onset (Andersson et al, 2000, 2009; Dornhoffer et al, 2006; Rossiter et al, 2006; Stevens et al, 2007; Heeren et al, 2014). The incidence of bilateral and unilateral tinnitus, duration of tinnitus exposure, and degree of distress were not routinely recorded.

\section{Influence of anxiety and depression}

Thirteen studies included measures of anxiety and/or depression and found a strong association between tinnitus, anxiety, depression, and somatic awareness which may have a confounding influence on cognitive performance (Table 2). Nine different measures were employed, with the Beck depression inventory (Beck et al, 1961) and the state-trait anxiety inventory (Speilberger et al, 1970) most frequently used at five instances each

\section{Influence of hearing loss}

Ten studies reported some audiometric data for their participants (Table 2), however, control subjects were only tested in 
Table 1. Aspects of cognition investigated by studies in the review.

\begin{tabular}{|c|c|c|c|c|}
\hline First author & Year & Aspect of cognition studied & Cognition evaluated using: & $\begin{array}{l}\text { Was cognition } \\
\text { worse with tinnitus }\end{array}$ \\
\hline Jacobson & 1996 & Selective Auditory attention & $\begin{array}{l}\text { ERPs (N1, P2, ND) \& Behavioural (Dichotic } \\
\text { auditory task) }\end{array}$ & Yes \\
\hline \multirow[t]{2}{*}{ Andersson } & 2000 & $\begin{array}{l}\text { Selective attention; executive processing; cognitive } \\
\text { flexibility; processing speed }\end{array}$ & Behavioural (Stroop colour word test) & Yes \\
\hline & 2002 & $\begin{array}{l}\text { Visual motor speed, selective attention, set shifting, } \\
\text { working memory }\end{array}$ & Behavioural (Digit-symbol test) & Yes \\
\hline Cuny & 2004 & Automatic attention & Behavioural (Attention capture task) & No \\
\hline Hallam & 2004 & Sharing of attention Sustained attention & $\begin{array}{l}\text { Behavioural (Bakan visual vigilance test, variable } \\
\text { fore-period reaction time, five choice serial } \\
\text { reaction time, delayed serial recall of digits, } \\
\text { CFQ) }\end{array}$ & Yes \\
\hline Andersson & 2005 & $\begin{array}{l}\text { Selective attention; executive processing abilities; cog- } \\
\text { nitive flexibility; Processing speed }\end{array}$ & Behavioural (emotional Stroop) & Yes \\
\hline Dornhoffer & 2006 & Attention Reaction time & ERPs \& Behavioural (Psychomotor vigilance task) & No \\
\hline Rossiter & 2006 & $\begin{array}{l}\text { Auditory verbal working memory, Visual divided } \\
\text { attention }\end{array}$ & Behavioural (reading span, CFQ) & Yes \\
\hline Stevens & 2007 & Sustained selective attention; divided attention & Behavioural (Stroop test) & Yes \\
\hline Delb & 2008 & Attention & ERPs (N100 amplitude, phase locking) & Yes \\
\hline Andersson & 2009 & Short term working memory, verbal ability & $\begin{array}{l}\text { Behavioural (serial recall test, Synomer cognitive } \\
\text { failures questionnaire) }\end{array}$ & No \\
\hline Muluk & 2010 & $\begin{array}{l}\text { Temporal and spatial orientation, short-term memory, } \\
\text { computation, } \\
\text { secondary memory, verbal attainment and con- } \\
\text { structive ability }\end{array}$ & Behavioural (MMSE) & No \\
\hline Gabr & 2012 & $\begin{array}{l}\text { Cognitive flexibility, visual-motor integration, visual } \\
\text { scanning and motor speed }\end{array}$ & $\begin{array}{l}\text { ERPs, Behavioural \& Oculomotor (MMSE, Trail } \\
\text { making tests, P300, smooth-pursuit, optokinetic, } \\
\text { gaze and saccadic eye movements) }\end{array}$ & Yes \\
\hline Alam & 2013 & Self-report no objective measure & CFQ & Yes \\
\hline Andersson & 2014 & Autobiographical memory & Behavioural (Autobiographical memory test) & Yes \\
\hline Paul & 2014 & Attention & $\begin{array}{l}\text { ERPs \& Behavioural (ASSR with push button } \\
\text { response) }\end{array}$ & No \\
\hline Heeren & 2014 & Alerting, orienting and executive control & Behavioural (Attention network test, MMT) & Yes \\
\hline Jackson & 2014 & $\begin{array}{l}\text { Selective attention; executive processing abilities; cog- } \\
\text { nitive flexibility; Processing speed }\end{array}$ & $\begin{array}{l}\text { Behavioural (Stroop test, Vienna determination } \\
\text { task) }\end{array}$ & Yes \\
\hline
\end{tabular}

ERP: Electrophysiological response, MMSE: Mini mental state examination, CFQ: Cognitive failures questionnaire.

seven studies. Additionally, self-report of normal hearing or selfcategorization of degree of hearing loss was reported by two studies (Rossiter et al, 2006; Andersson et al, 2009). Two studies measured hearing thresholds for some but not all of their clinical groups (Hallam et al, 2004; Andersson et al, 2013). Only Cuny et al (2004) matched their subject and control groups for hearing thresholds and found that tinnitus disturbed attention shifting. Five studies did not report assessing hearing thresholds (Andersson et al, 2000, 2005; Dornhoffer et al, 2006; Heeren et al, 2014; Jackson et al, 2014). Of the remaining six studies, Gabr et al (2011) failed to discuss the impact of hearing loss on cognition when concluding that the tinnitus group had an overall cognitive impairment, and five did not report assessing hearing thresholds.

\section{Effect of masking}

Two studies investigated the impact of masking tinnitus on cognitive function. Andersson (2002) found that tinnitus subjects performed more poorly than control subjects in all conditions. They also found that continuous masking had no impact upon the results whereas intermittent masking negatively impacted both the control and tinnitus subjects. Andersson et al (2009) found no difference in performance between tinnitus and control subjects and no difference between constant and intermittent masking.

\section{Simulated tinnitus}

One study used a sound designed to mimic tinnitus to investigate the impact of simulated tinnitus upon cognitive function (Cuny et al, 2004), reporting that subjects with intrinsic unilateral tinnitus responded with greater accuracy on an oddball paradigm categorization task when the deviant stimuli was presented to their tinnitus ear, compared to their non-tinnitus ear. No difference was noted amongst subjects with simulated tinnitus.

\section{Relationship between tinnitus and cognition}

Nine studies implicated negative effects of tinnitus upon attention (Table 1). Six studies found general/nonspecific cognitive impairment amongst tinnitus groups compared to control groups. Three found no specific difference in cognitive function between tinnitus and control subjects. One study reported that the tinnitus group's mini mental state examination (MMSE) (Folstein et al, 1975) scores were within normal limits but significantly lower than 
Table 2. Descriptive overview of studies evaluating the effects of tinnitus upon cognition.

\begin{tabular}{|c|c|c|c|c|c|c|}
\hline First author & Year & $\begin{array}{l}\text { Control group } \\
\text { matched for age }\end{array}$ & $\begin{array}{l}\text { Control group matched } \\
\text { for hearing loss }\end{array}$ & $\begin{array}{c}\text { Tinnitus descriptions/ } \\
\text { classification }\end{array}$ & Tinnitus evaluated & $\begin{array}{c}\text { Anxiety \& } \\
\text { depression evaluated }\end{array}$ \\
\hline Jacobson & 1996 & $x$ & $x$ & $\checkmark$ & $x$ & $x$ \\
\hline Andersson & 2000 & $\checkmark$ & $x$ & $\checkmark$ & $\checkmark$ & $\checkmark$ \\
\hline Andersson & 2002 & $x$ & $x$ & $\checkmark$ & $\checkmark$ & $\checkmark$ \\
\hline Cuny & 2004 & $x$ & $x$ & $\checkmark$ & $\checkmark$ & $x$ \\
\hline Hallam & 2004 & $x$ & $x$ & $\checkmark$ & $x$ & $\checkmark$ \\
\hline Andersson & 2005 & $x$ & $x$ & $x$ & $\checkmark$ & $\checkmark$ \\
\hline Stevens & 2007 & $\checkmark$ & $x$ & $\checkmark$ & $\checkmark$ & $\checkmark$ \\
\hline Delb & 2008 & $x$ & $x$ & $\checkmark$ & $\checkmark$ & $\checkmark$ \\
\hline Andersson & 2009 & $\checkmark$ & & $\checkmark$ & $\checkmark$ & $\checkmark$ \\
\hline Muluk & 2010 & $x$ & $x$ & $\checkmark$ & $\checkmark$ & $x$ \\
\hline Gabr & 2012 & $x$ & $x$ & $x$ & $x$ & $\checkmark$ \\
\hline Alam & 2013 & $\checkmark$ & $x$ & $\checkmark$ & $\checkmark$ & $\checkmark$ \\
\hline
\end{tabular}

the control group. Two studies highlighted an impact on cognitive function associated with tinnitus distress.

\section{Discussion}

In clinical settings, reports of cognitive difficulties are common amongst tinnitus sufferers (Andersson et al, 2009; Pierce et al, 2012; Searchfield, 2014). The present study unveiled a complex and multifaceted relationship between tinnitus and cognitive function. Arriving at a concise conclusion regarding the impact of tinnitus upon cognitive function is complicated by the heterogeneity of the studies undertaken. Additionally, understanding regarding cognitive function and the possible impact of concurrent conditions continues to evolve and both the design and conclusions of the studies in this review reflect that evolution.

\section{Cohort descriptions}

Across the studies, tinnitus and control groups were often not matched for age or hearing loss, despite both factors having been reported as impacting cognitive function (Lin et al, 2004, 2011; Jorgensen et al, 2014; Dupuis et al, 2015). Influence from confounding factors such as degree of anxiety, depression, and education levels were also often not well controlled. It is possible that variables other than tinnitus may have contributed to the findings and compromised the conclusions drawn.

\section{Measures of cognition and electrophysiological indices}

Several cognitive tests were employed and the variety of tests and study designs may have contributed to the differing conclusions reached. However, the sensitivity of the cognitive tests was poorly accounted for, as was the potential for interaction between neural sub-networks to influence results. Similarly, although, highly varied levels of cognitive difficulties have been reported clinically, the studies examined in this review failed to sufficiently explore these variations and the clinical application of the studies' results is limited (Andersson, 2002; Dornhoffer et al, 2006; Muluk et al,
2010). Increased use of study designs combining neurophysiological assessments, neuroimaging, and behavioural tests of cognitive function may assist to expand our understanding of how, why, and the extent to which tinnitus impacts attention, cognitive processing, and memory.

ERPs provide an objective measure of both voluntary selective attention and involuntary attention and are less subject to subjective influences such as mood and memory than behavioural tests (Jacobson et al, 1996; Cuny et al, 2004). In several studies ERP results supported the behavioural cognitive tests, showing that tinnitus adversely affects auditory attention (Jacobson et al, 1996; Delb et al, 2008; Paul et al, 2014). However, one study found that although the delayed mean P300 latency of the tinnitus subjects supported a finding of impaired cognitive function, the behavioural findings of the cognition screening scores were within normal limits (Gabr et al, 2011).

Determining which cognitive function tests are most suitable for testing which aspects of cognitive function, quantify the degree of impact, and are least influenced by the accompanying sensory impairments or medical issues is challenging (Andersson, 2002; Gates et al, 2010; Dupuis et al, 2015).

\section{Measures of tinnitus and tinnitus descriptions}

Significant variation was also noted in the methods used to describe and measure tinnitus. Gabr et al, (2011) neither described nor measured the tinnitus experienced, whereas, Andersson et al (2000) and Paul et al (2014) provided extensive tinnitus descriptions and used validated self-report tinnitus distress scales. Such variation creates difficulties when comparing studies or formulating a global conclusion regarding whether tinnitus distress or duration impacts the relationship between tinnitus and cognitive function.

\section{Influence of anxiety and depression}

Both anxiety and depression negatively influence information processing (Broadbent et al, 1986; Andersson et al, 2005). Anxiety disorders produce a cognitive bias during the early stages 
of information processing, whilst depression influences the later stages of information processing (Broadbent et al, 1986; Andersson et al, 2005; Stevens et al, 2007). Studies which have not screened for anxiety or depression may incorrectly ascribe cognitive difficulties to tinnitus. Stevens et al (2007) noted that people with invasive tinnitus tend to be high 'self-attenders', more predisposed to attending to somatic or internal sensations, beliefs and thoughts. Subsequently they may have heightened sensitivity to cognitive failings and more predisposed to remember and report each perceived failing due to cognitive bias (Hallam et al, 2004; Delb et al, 2008; Andersson et al, 2013).

\section{Influence of hearing loss}

Hearing loss was not well accounted for by the studies reviewed despite the known strong associations between tinnitus and hearing loss, and impact of hearing loss upon cognitive function (Rabbitt, 1991; Cuny et al, 2004; Tun et al, 2009; Baldwin \& Ash, 2011; Dupuis, et al, 2015). A hearing loss of $25 \mathrm{~dB}$ is suggested to have the equivalent effect on cognitive function as ageing seven years (Lin et al, 2011). Additionally, people with hearing loss will wait an average of ten years before seeking treatment for their hearing loss; this may exacerbate the cognitive difficulties of a person with both hearing loss and invasive tinnitus (Davis et al, 2007). When hearing levels are not quantified or when hearing levels are not matched it becomes difficult to determine whether the cognitive effects noted are attributable to tinnitus or hearing loss (Rabbitt, 1991; Andersson et al, 2000; Dupuis et al, 2015).

\section{Effect of masking}

Andersson (2002) and Andersson et al (2009) both investigated the impact of masking tinnitus on cognitive function and reached differing conclusions. Andersson et al (2002) found that intermittent masking disrupted cognitive function in both the control and tinnitus subjects and also that the tinnitus subjects performed more poorly in general. However, Andersson et al (2009) found no influence of continuous or intermittent masking upon a task of serial recall in either tinnitus or control subjects and no behaviourally measurable difference between the two groups. The results of these studies may influence treatment recommendations because masking is commonly used therapeutically for tinnitus. The issue of masking also raises a possible relationship between tinnitus and central auditory processing difficulties and the potential for an adverse influence upon tests of cognitive function (Searchfield et al, 2007; Gates et al, 2010).

\section{Simulated tinnitus}

Differences in how individuals respond to intrinsic tinnitus compared to external simulated tinnitus was highlighted by Cuny et al (2004) who showed that continuous simulated tinnitus failed to invoke the same automatic attention capture and direction effects as intrinsic tinnitus. Dornhoffer et al (2006) suggested that the different habituation responses noted in tinnitus patients to externally generated sounds compared to tinnitus may be associated with corresponding differences in thalamo-cortical activation. External sounds invoke bilateral activation whereas intrinsic tinnitus invokes a predominantly unilateral activation. This asymmetrical activation may contribute to disturbances in sustained attention and explain why some people habituate to external noises but not intrinsic tinnitus (Dornhoffer et al, 2006).

\section{Relationship between tinnitus and cognition}

Understanding of cognitive function, the various elements of cognitive function, the interrelationships between them, and how to test them and interpret results continues to evolve (Dupuis et al, 2015). Accordingly, differences between the earlier and recent studies reflect this development with conclusions and interpretations progressing from general to specific. Many earlier studies such as Jacobson et al (1996) and Andersson et al (2000) found general and nonspecific cognitive impairment in tinnitus subjects compared to healthy controls. More recent studies implicated an impact upon the executive control of attention with levels of anxiety, depression, and somatic sensitivity responsible for much variability (Dornhoffer et al, 2006; Andersson et al, 2013; Heeren et al, 2014; Jackson et al, 2014). This suggests that people with invasive tinnitus have trouble with allocation of attentional resources and resolving conflict between responses. However, the quantifiable impact may be minor or subtle (Rossiter et al, 2006; Gabr et al, 2011). As such when tasks are demanding or challenging, a person with invasive tinnitus may perform well and only experience difficulties when tasks are less demanding.

\section{Clinical implications}

The relationship between impaired cognitive function, tinnitus, anxiety and depression, and hearing loss were supported by the findings of the studies in this review and have implications for the successful management of people with invasive tinnitus (Gabr et al, 2011).

Both depression and anxiety have a compounding effect on poor quality of life, health, mood, and cognitive flexibility which may in turn compromise individuals' ability to comply with recommended management strategies (Gabr et al, 2011). Jackson et al (2014) recognized the negative effects of anxiety on cognitive function in those experiencing tinnitus, and recommended reducing anxiety to improve cognitive performance.

Treatment programs incorporating factual informational counselling (Tunkel et al, 2014) and attention training (Searchfield et al, 2007; Delb et al, 2008; Heeren et al, 2014) have been shown to reduce tinnitus distress and perceived cognitive difficulties. Informational counselling and cognitive behavioural therapy should be tailored to each patient's specific concerns and encompass executive control of attention as well as the additive effect of anxiety, depression, and hearing loss (Tyler \& Baker, 1983; McKenna et al, 1995; Muluk et al, 2010; Tunkel et al, 2014). Given the strong association between tinnitus and hearing loss and the impact of hearing loss upon cognitive function, counselling should encourage patients with both tinnitus and hearing loss to pursue hearing loss management. Cognitive behavioural therapy and mindfulness can be used to assist people with invasive tinnitus direct their attention away from their tinnitus (Heeren et al, 2014; Hoare et al, 2014). Targeted attention training can specifically improve aspects of attention (Searchfield et al, 2007; Heeren et al, 2014; Hoare et al, 2014).

Incorporating a cognitive screening test into the assessment for those people with invasive tinnitus reporting cognitive difficulties may be beneficial by either confirming the difficulties reported or allaying their concerns and providing a benchmark for future improvements. Using a cognitive screening test that is sensitive to the impact of tinnitus, such as the trail making test (Reitan, 1958) would allow clinicians to validate their patients' reported 
difficulties as well as track any changes in perceived cognitive difficulties and tinnitus distress over the course of treatment (Sweet et al, 2011; Dupuis et al, 2015).

\section{Limitations and directions for future study}

Although a rigorous search strategy was employed, this review specifically excluded studies not published in English. The findings of this study were confounded by the lack of clear reporting of tinnitus descriptions, duration control group matching for hearing loss, anxiety, and depression.

Subsequently, it is recommended that future studies draw from the experience of the field of cognitive psychology, especially from studies investigating emotional disorders such as chronic pain and anxiety where attentional biases can impact upon cognition (Andersson et al, 2005), and from studies exploring the relationship between hearing loss and cognition (Taljaard et al, 2016). Future studies exploring the relationship between tinnitus and cognition need to employ strategies that evaluate cognitive function, and control for, or account for, confounding variables. It is suggested that studies focusing on the impact of tinnitus on cognitive function should: (1) Employ standardized neurocognitive tests both verbal and non-verbal that evaluate multiple cognitive domains and include tests for attention and executive processing, (2) Use a thorough standardized approach to tinnitus assessment in terms of description and evaluation including type, duration, and location, distress levels at time of testing, and psychoacoustic measures, (3) Control for the impact of potentially confounding comorbid medical conditions such as depression, anxiety age, and somatic sensitivity, (4) Reduce potential impact from sensory impairment by ensuring subjects have access to both visual and auditory test stimuli (Dupuis et al, 2015), and (5) Reduce the potential for impact from environmental factors by ensuring that test conditions are well-described and that testing is undertaken in well-lit, quiet conditions where potential distractions are minimal (Dupuis et al, 2015).

\section{Conclusion}

The significant heterogeneity of study designs, cognitive tests, subject characteristics, and management of covariables in the 18 studies reviewed makes it difficult to compare and contrast the studies directly. Accumulatively, evidence suggests that the cognitive difficulties reported by people with invasive tinnitus relate to an impact of tinnitus upon the executive control of attention. The studies also highlight the reciprocal relationship between cognitive function and tinnitus with the potential for concomitant problems such as depression, anxiety, somatic sensitivity, and hearing loss to compound the cognitive difficulties noted. This review highlights the need to further explore contributions from hearing loss, age, anxiety, and depression. It also highlights the need to explore effective management strategies.

Declaration of interest: The authors of this paper declare no conflict of interest or competing financial interests.

\section{Acknowledgements}

The authors would like to acknowledge the support of the Ear Science Institute Australia and the Lions Hearing Clinic.

\section{References}

Alam N., Katarkar A., Shah P., Jalvi R., Jain A. et al. 2012. Audiological, psychological and cognitive characteristics of tinnitus sufferers. Indian $J$ Otol, 18, 20-23.

Andersson G. 2002. Psychological aspects of tinnitus and the application of cognitive-behavioral therapy. Clin Psychol Rev, 22, 977-990.

Andersson G., Bakhsh R., Johansson L., Kaldo V. \& Carlbring P. 2005. Stroop facilitation in tinnitus patients: an experiment conducted via the world wide web. Cyberpsychol Behav, 8, 32-38.

Andersson G., Edsjö L., Kaldo V. \& Westin V. 2009. Tinnitus and shortterm serial recall in stable versus intermittent masking conditions. Scand J Psychol, 50, 517-522.

Andersson G., Eriksson J., Lundh L.G. \& Lyttkens L. 2000. Tinnitus and cognitive interference: a stroop paradigm study. J Speech Lang Hear Res, 43, 1168-1173.

Andersson G., Hesser H., Cima R.F. \& Weise C. 2013. Autobiographical memory specificity in patients with tinnitus versus patients with depression and normal controls. Cogn Behav Ther, 42, 116-126.

Andersson G. \& McKenna L. 2006. The role of cognition in tinnitus. Acta OtoLaryngol, 126, 39-43.

Araneda R., De Volder A.G., Deggouj N., Philippot P., Heeren A. et al. 2015. Altered top-down cognitive control and auditory processing in tinnitus: Evidences from auditory and visual spatial stroop. Restor Neurol Neurosci, 33, 67-80.

Baldwin C.L. \& Ash I.K. 2011. Impact of sensory acuity on auditory working memory span in young and older adults. Psychol Aging, 26, 8591.

Beck A.T., Ward C. \& Mendelson M. 1961. Beck depression inventory (BDI). Arch Gen Psychiatry, 4, 561-571.

Broadbent D.E., Broadbent M.H. \& Jones J.L. 1986. Performance correlates of self-reported cognitive failure and of obsessionality. $\mathrm{Br} \mathrm{J}$ Clin Psychol, 25, 285-299.

Coleman K., Norris S., Weston A., Grimmer-Somers K., Hillier S. et al. 2005. NHMRC Additional Levels of Evidence and Grades for Recommendations for Developers of Guidelines. Canberra: NHMRC.

Cuny C., Norena A., El Massioui F. \& Chéry-Croze S. 2004. Reduced attention shift in response to auditory changes in subjects with tinnitus. Audiol Neurootol, 9, 294-302.

Davis A., Smith P., Ferguson M., Stephens D. \& Gianopoulos I. 2007. Acceptability, Benefit and Costs of Early Screening for Hearing Disability: A Study of Potential Screening Tests and Models: National Coordinating Centre for Health Technology Assessment, Southampton: University of Southampton.

De Ridder D., Elgoyhen A.B., Romo R. \& Langguth B. 2011. Phantom percepts: Tinnitus and pain as persisting aversive memory networks. Proc Natl Acad Sci USA, 108, 8075-8080.

De Ridder D., Vanneste S., Weisz N., Londero A., Schlee W. et al. 2014. An integrative model of auditory phantom perception: Tinnitus as a unified percept of interacting separable subnetworks. Neurosci Biobehav Rev, 44, 16-32.

Delb W., Strauss D.J., Low Y.F., Seidler H., Rheinschmitt A. et al. 2008. Alterations in event related potentials (ERP) associated with tinnitus distress and attention. Appl Psychophysiol Biofeedback, 33, 211-221.

Dornhoffer J., Danner C., Mennemeier M., Blake D. \& Garcia-Rill E. 2006. Arousal and attention deficits in patients with tinnitus. Int Tinnitus J, 12, 9-16.

Dupuis K., Pichora-Fuller M.K., Chasteen A.L., Marchuk V., Singh G. et al. 2015. Effects of hearing and vision impairments on the Montreal cognitive assessment. Aging Neuropsychol Cogn, 22, 413-437.

Folstein M.F., Folstein S.E. \& McHugh P.R. 1975. "Mini-mental state'”: a practical method for grading the cognitive state of patients for the clinician. J Psychiatr Res, 12, 189-198.

Gabr T.A., El-Hay M.A. \& Badawy A. 2011. Electrophysiological and psychological studies in tinnitus. Auris Nasus Larynx, 38, 678-683. 
Gates G.A., Gibbons L.E., McCurry S.M., Crane P.K., Feeney M.P. et al. 2010. Executive dysfunction and presbycusis in older persons with and without memory loss and dementia. Cogn Behav Neurol, 23, 218-223.

Halford J.B. \& Anderson S.D. 1991. Tinnitus severity measured by a subjective scale, audiometry and clinical judgement. J Laryngol Otol, $105,89-93$

Hall D.A., Láinez M.J., Newman C.W., Sanchez T., Egler M. et al. 2011. Treatment options for subjective tinnitus: self reports from a sample of general practitioners and ENT physicians within Europe and the USA. BMC Health Serv Res, 11, 1. DOI: 10.1186/1472-6963-11-302.

Hallam R. 1996. Manual of the Tinnitus Questionnaire (TQ). London: Psychological Corporation.

Hallam R., McKenna L. \& Shurlock L. 2004. Tinnitus impairs cognitive efficiency. Int $J$ Audiol, 43, 218-226.

Heeren A., Maurage P., Perrot H., De Volder A., Renier L. et al. 2014. Tinnitus specifically alters the top-down executive control sub-component of attention: Evidence from the attention network task. Behav Brain Res, 269, 147-154.

Hoare D.J., Edmondson-Jones M., Gander P.E. \& Hall D.A. 2014. Agreement and reliability of tinnitus loudness matching and pitch likeness rating. PloS One, 9. DOI:10.1371/journal.pone.0114553.

Jackson J.G., Coyne I.J. \& Clough P.J. 2014. A preliminary investigation of potential cognitive performance decrements in non-help-seeking tinnitus sufferers. Int $J$ Aud, 53, 88-93.

Jacobson G.P., Calder J.A., Newman C.W., Peterson E.L., Wharton J.A. et al. 1996. Electrophysiological indices of selective auditory attention in subjects with and without tinnitus. Hear Res, 97, 66-74.

Jorgensen L., Palmer C. \& Fischer G. 2014. Evaluation of hearing status at the time of dementia diagnosis. Audiology Today, 26, 38-32.

Lanting C., De Kleine E. \& Van Dijk P. 2009. Neural activity underlying tinnitus generation: results from PET and fMRI. Hear Res, 255, 1-13.

Liberati A., Altman D.G., Tetzlaff J., Mulrow C., Gøtzsche P.C. et al. 2009. The PRISMA statement for reporting systematic reviews and metaanalyses of studies that evaluate health care interventions: Explanation and elaboration. Ann Int Med, 151, W-65-W-94.

Lin F.R., Ferrucci L., Metter E.J., An Y., Zonderman A.B. et al. 2011. Hearing loss and cognition in the Baltimore longitudinal study of aging. Neuropsychology, 25, 763

Lin F.R., Metter E.J., O’brien R.J., Resnick S.M., Zonderman A.B. et al. 2011. Hearing loss and incident dementia. Arch Neurol, 68, 214-220.

Lin M.Y., Gutierrez P.R., Stone K.L., Yaffe K., Ensrud K.E. et al. 2004. Vision impairment and combined vision and hearing impairment predict cognitive and functional decline in older women. J Am Geriatr Soc, 52, 1996-2002.

Mahoney C.J., Rohrer J.D., Goll J.C., Fox N.C., Rossor M.N. et al. 2011. Structural neuroanatomy of tinnitus and hyperacusis in semantic dementia. J Neurol Neurosurg Psychiatr, 82, 1274-1278.

McCormack A., Edmondson-Jones M., Fortnum H., Dawes P., Middleton H. et al. 2014. The prevalence of tinnitus and the relationship with neuroticism in a middle-aged UK population. J Psychosom Res, 76, $56-60$.

McKenna L., Hallam R. \& Shurlock L. 1995. Cognitive Functioning in Tinnitus Patients Proceedings of the Fifth International Tinnitus Seminar: American Tinnitus Association Portland, Oregon, pp. 589-595.

Melcher J., Sigalovsky I., Guinan J. \& Levine R. 2000. Lateralized tinnitus studied with functional magnetic resonance imaging: Abnormal inferior colliculus activation. J Neurophysiol, 83, 1058-1072.

Mohamad N., Hoare D.J. \& Hall D.A. 2016. The consequences of tinnitus and tinnitus severity on cognition: A review of the behavioural evidence. Hear Res, 332, 199-209.

Moher D., Liberati A., Tetzlaff J. \& Altman D.G. 2009. Preferred reporting items for systematic reviews and meta-analyses: The PRISMA statement. Ann Intern Med, 151, 264-269.

Mokkink L.B., Terwee C.B., Stratford P.W., Alonso J., Patrick D.L. et al. 2009. Evaluation of the methodological quality of systematic reviews of health status measurement instruments. Qual Life Res, 18, 313-333.
Mulders W. \& Robertson D. 2009. Hyperactivity in the auditory midbrain after acoustic trauma: dependence on cochlear activity. Neuroscience, 164, 733-746.

Muluk N., Tuna E. \& Arikan O. 2010. Effects of subjective tinnitus on sleep quality and mini mental status examination scores. B-ENT, 6, 271-280.

Pace E. \& Zhang J. 2013. Noise-induced tinnitus using individualized gap detection analysis and its relationship with hyperacusis, anxiety, and spatial cognition. PLoS One, 8, e75011.

Panic N., Leoncini E., de Belvis G., Ricciardi W. \& Boccia S. 2013. Evaluation of the endorsement of the preferred reporting items for systematic reviews and meta-analysis (PRISMA) statement on the quality of published systematic review and meta-analyses. PloS One, 8, e83138.

Paul B.T., Bruce I.C., Bosnyak D.J., Thompson D.C. \& Roberts L.E. 2014. Modulation of electrocortical brain activity by attention in individuals with and without tinnitus. Neural Plast, 2014, http://dx.doi.org/10/1155/ 2014/127824.

Pierce K.J., Kallogjeri D., Piccirillo J.F., Garcia K.S., Nicklaus J.E. et al. 2012. Effects of severe bothersome tinnitus on cognitive function measured with standardized tests. J Clin Exp Neuropsychol, 34, 126-134.

Potashner S., Suneja S. \& Benson C. 1997. Regulation ofd-aspartate release and uptake in adult brain stem auditory nuclei after unilateral middle ear ossicle removal and cochlear ablation. Exp Neurol, 148, 222-235.

Rabbitt P. 1991. Mild hearing loss can cause apparent memory failures which increase with age and reduce with IQ. Acta Otolaryngol, 111, 167-176.

Rauschecker J.P., Leaver A.M. \& Mühlau M. 2010. Tuning out the noise: limbic-auditory interactions in tinnitus. Neuron, 66, 819-826.

Reitan R.M. 1958. Validity of the trail making test as an indicator of organic brain damage. Percept Mot Skills, 8, 271-276.

Rossiter S., Stevens C. \& Walker G. 2006. Tinnitus and its effect on working memory and attention. $J$ Speech Lang Hear Res, 49, 150-160.

Searchfield G.D. 2014. Tinnitus what and where: An ecological framework. Front Neurol, 5. DOI:10.3389/fneur.2014.00271.

Searchfield G.D., Morrison-Low J. \& Wise K. 2007. Object identification and attention training for treating tinnitus. Prog Brain Res, 166, 441-460.

Shulman A., Strashun A., Afriyie M., Aronson F., Abel W. et al. 1994. SPECT Imaging of Brain and Tinnitus-Neurotologic/Neurologic Implications. Int Tinnitus J, 1, 13-29.

Speilberger C.D., Gorsuch R.L. \& Lushe R.E. 1970. Test Manual for the stateTrait Anxiety Inventory. Palo Alto, CA: Consulting Psychologists Press.

Stevens C., Walker G., Boyer M. \& Gallagher M. 2007. Severe tinnitus and its effect on selective and divided attention: acufeno severo y sus efectos sobre la atención selectiva y dividida. Int $J$ Audiol, 46, 208-216.

Sweet L., Van Adel M., Metcalf V., Wright L., Harley A. et al. 2011. The Montreal cognitive assessment (MoCA) in geriatric rehabilitation: Psychometric properties and association with rehabilitation outcomes. Int Psychogeriatr, 23, 1582-1591.

Taljaard D., Olaithe M., Brennan-Jones C.G., Eikelboom R.H. \& Buck R.S. 2016. The relationship between hearing impairment and cognitive function: A meta analysis. Clin Otolaryngol, Online 28 February 2016. [Epub ahead of print]. DOI:10.111/coa.12607

Tun P.A., McCoy S. \& Wingfield A. 2009. Aging, hearing acuity, and the attentional costs of effortful listening. Psychol Aging, 24, 761-766.

Tunkel D.E., Bauer C.A., Sun G.H., Rosenfeld R.M., Chandrasekhar S.S. et al. 2014. Clinical Practice guideline: Tinnitus. Otolaryngol Head Neck Surg, 151, S1-S40.

Tyler R.S. \& Baker L.J. 1983. Difficulties experienced by tinnitus sufferers. J Speech Hear Disord, 48, 150-154.

Vogler D.P., Robertson D. \& Mulders W.H. 2011. Hyperactivity in the ventral cochlear nucleus after cochlear trauma. J Neurosci, 31, 6639-6645.

Westin V., Hayes S.C. \& Andersson G. 2008. Is it the sound or your relationship to it? The role of acceptance in predicting tinnitus impact. Behav Res Ther, 46, 1259-1265.

Wilson P.H., Henry J., Bowen M. \& Haralambous G. 1991. Tinnitus reaction questionnaire: Psychometric properties of a measure of distress associated with tinnitus. $J$ Speech Hear Res, 34, 197-201. 